\title{
P04-3-17 Poster session
}

\section{CP-25, a modified compound from paeoniflorin, alleviated collagen-induced arthritis, which is associated with suppressing immune response and synovium inflammation}

\author{
Jingyu Chen, Ying Wang, Hua-Xun Wu, Shang-Xue Yan, Yan Chang, Wei Wei
}

Key Laboratory of Anti-inflammatory and Immune Medicine, Ministry of Education, China, Institute of Clinical Pharmacology of Anhui Medical University, China

Background: CP-25 (paeoniflorin-6-O-benzene sulfonate, patent number in China, ZL201210030616.4) is a modified compound from paeoniflorin (Pae), the main bioactive components of the total glucosides of peony (TGP). In current study, we evaluated the effect of CP-25 focused on T cell-dependent immune response and synoviocytes activation in CIA mice, and assayed beta 2-AR signaling on immune cells and synoviocytes to find the potential mechanism which was responsible for the anti-inflammatory and immunoregulatory effect of CP-25 in RA. Methods: Collagen type 2 was used to induce CIA mice. After the onset of arthritis, mice were given CP-25 (17.5, 35, and $70 \mathrm{mg} / \mathrm{kg})$ or MTX (2.0 mg/kg). To evaluate the severity of arthritis, arthritis index and swollen joint count were evaluated every 3 days. Histopathology of joint and spleen were assayed. Subsets of $\mathrm{T}$ cells including CD4+CD62L+ (naive T cells), CD4+CD25+ (activated T cells), Th1, Th17, Tfh and CD19+CD138+ plasma B cells in the spleen of CIA mice were assayed by flow cytometry. Proliferation of T cells, B cells and synoviocytes were evaluated by CCK. The expression of beta 2-AR was assayed by flow cytometry, immunohistochemical staining and western blot. Synoviocytes migration and invasion were assayed by transwell. Results: Administration of CP-25 $(35,70 \mathrm{mg} / \mathrm{kg})$ significantly attenuated arthritis index and swollen joint counts, restored the histopathological change of joint and spleen. CP-25 (35, 70mg/kg) suppressed T cells and B cells proliferation, up-regulated the percentage of naive T cells, down-regulated the percentage of activated T cells, Th1, Th17, Tfh and CD19+CD138+ plasma B cells in the spleen of CIA mice. In vivo, CP-25 up-regulated the membrane expression of beta 2-AR on T cells, B cells, DCs and in synovium from CIA mice. In vitro, beta 2-AR agonists (salbutamol) had no effect on RA synoviocytes proliferation, migration, and invasion, but treatment with CP-25 and salbutamol could suppresse proliferation, migration, and invasion of RA synoviocytes. CP-25 up-regulated beta 2-AR membrane expression and down-regulated GRK2 membrane expression on synoviocytes. Conclusions: Our results suggest that CP-25 suppressed $\mathrm{T}$-dependent immune response and synovium inflammation in CIA, which is associated with promoting beta 2-AR signaling by reducing beta 2-AR desensitization. 